\title{
Safety and efficacy of nitric oxide in chronic lung disease
}

\section{P L Clark, I I Ekekezie, H A Kaftan, C A Castor, W E Truog}

Arch Dis Child Fetal Neonatal Ed 2002;86:F41-F45

See end of article for authors' affiliations

Correspondence to: Dr Truog, Department of Pediatrics, University of Missouri-Kansas School of Medicine, Kansas City, USA:

wtruog@cmh.edu

Accepted 23 August 2001

\begin{abstract}
Background: Therapies for neonatal chronic lung disease (CLD) of prematurity have had limited success.

Aims: To determine whether inhaled nitric oxide (INO) administered to very low birthweight infants with developing CLD might improve oxygenation without adverse effects.

Methods: Subjects were 10-30 days of age, birth weight $<1250 \mathrm{~g}$, with developing or established $\mathrm{CLD}$, and requiring mechanical ventilation with mean airway pressure $\geqslant 7 \mathrm{~cm} \mathrm{H}_{2} \mathrm{O}$ and $\mathrm{FlO}_{2} \geqslant 0.40$. We monitored changes in oxygenation and $\mathrm{FIO}_{2}$ requirement during treatment with INO (initial dose 20 ppm). Tracheal aspirate samples obtained before, during, and after treatment were analysed for interleukin $1 \beta(\mathrm{IL}-1 \beta)$, IL-8, 8-epi-prostaglandin $\mathrm{F}_{2 \alpha}\left(8\right.$-epi-PGF $\left.\mathrm{e}_{2 \alpha}\right)$, laminin, and endothelin 1 (ET-1) to assess any potential effects of INO on markers of inflammation peroxidation, basement membrane injury, or vasoactivity.

Results: Thirty three patients met entry criteria. Mean gestational age was 25 (SD 2) weeks; birth weight was 736 (190 g); age of study infants was 19 (6) days (range 9-29). Mean $\mathrm{FlO}_{2}$ decreased from baseline $(0.75)$ to 0.58 at 72 hours. Duration of therapy was seven days. Tracheal aspirate concentrations of IL-1 $\beta$, IL-8, 8-epi-PGF ${ }_{2 \alpha}$ ET-1, and laminin were unchanged between baseline and 48 hours of INO, and 48 hours after discontinuation of INO. No new cases of, nor extension of, intraventricular haemorrhage occurred. Four infants died.

Conclusion: INO ( $\leqslant 20 \mathrm{ppm}$ ) improved oxygenation in most infants with early CLD, without inducing changes in markers of inflammatory or oxidative injury.
\end{abstract}

$\mathrm{N}$ eonatal chronic lung disease (CLD), defined as the continuing need in preterm infants for supplemental inspired oxygen at 36 weeks postconceptional age, affects an estimated 12000 infants per year in the USA ${ }^{1}$ and many more infants worldwide. Limitations of current treatments for CLD have prompted a search for other interventions. One potentially useful therapy, on the basis of its known effectiveness to improve oxygenation in near term neonates with hypoxic respiratory failure, is inhaled nitric oxide (INO). No reports, to our knowledge, describe INO use in extremely preterm infants beginning at 10 days of age, when signs of CLD are becoming apparent.

The impact of INO, administered for a clinically meaningful time, on an already inflamed, immaturely developed lung, is largely unknown. Inflammation contributes to CLD, and nitric oxide can have both proinflammatory and anti-inflammatory effects. In the presence of high $\mathrm{FIO}_{2}$, $\mathrm{NO}$ is converted to $\mathrm{NO}_{2}$, peroxynitrite, and other oxides of nitrogen, which may initiate or exacerbate pulmonary inflammation. However, nitric oxide may modulate the pulmonary inflammatory response by downregulating the production of inflammatory cytokines, ${ }^{2}$ and by decreasing lung neutrophil accumulation. ${ }^{3}$

Because we considered it premature to conduct a prospective randomised trial of INO in the very low birthweight (VLBW) infant population with early CLD, based on the limited data available regarding dosage, efficacy, and especially safety, we conducted an open trial of INO in CLD, emphasising safety issues and short term efficacy in a severely ill group of patients.

\section{METHODS}

The Pediatric Institutional Review Board of the University of Missouri-Kansas City and the Children's Mercy Hospital approved the study protocol.

\section{Subjects}

All patients admitted to the Intensive Care Nursery from June 1997 to June 1999 were reviewed for eligibility. Parental con- sent was obtained for all eligible candidates identified, with no parental refusals. Inclusion criteria included birth weight $<1250$ g; age $<30$ days, but $\geqslant 10$ days; need for assisted ventilation; need for $\mathrm{FIO}_{2} \geqslant 0.4$ without fluctuations of $>0.25$ in the preceding 24 hours; and clinical course and radiographic findings compatible with CLD.

Exclusion criteria included: initiation of systemic corticosteroid or inhaled $\beta$ agonist therapy within the preceding 48 hours; new diagnosis of sepsis (two blood cultures yielding growth of a single pathogenic organism) within the preceding 48 hours; thrombocytopenia $\left(<100000 / \mathrm{mm}^{3}\right)$; progressive intraventricular haemorrhage; obviously lethal congenital anomaly; and complex congenital heart disease. The clinical course and chest radiographs of potential subjects were reviewed prior to enrolment by at least two investigators not serving as the patient's attending physician.

\section{Intervention}

Inhaled nitric oxide was administered initially at $20 \mathrm{ppm}$. Arterial oxygen saturation was monitored by pulse oximetry, and transcutaneous levels of $\mathrm{CO}_{2}$ and $\mathrm{O}_{2}$ were measured over the next three hours to ascertain response. $\mathrm{FIO}_{2}$ was adjusted to keep $\mathrm{SpO}_{2}$ at $89-96 \%$. No change in mean airway pressure occurred during this period. At the end of the initial three hour treatment period, a decision was made by the investigators regarding continuation of INO. If there was evidence of efficacy $\left(\mathrm{FIO}_{2}\right.$ decreased by $\geqslant 0.10, \mathrm{SpO}_{2}$ increased by $\geqslant 10 \%$, or $\mathrm{TcPO}_{2}$ improved by $1.3 \mathrm{kPa}$ ), INO was continued at $20 \mathrm{ppm}$.

Abbreviations: CLD, chronic lung disease; $C M V$, conventional mechanical ventilation; ELISA, enzyme linked immunoassay; HFOV, high frequency oscillatory ventilation; INO, inhaled nitric oxide; IVH, intraventricular haemorrhages; PCA, postconceptional age; PVR, pulmonary vascular resistance; sSC-lgA, soluble secretory component of $\lg A$; VLBW, very low birthweight 
After 36 hours, the dose of INO was reduced to $15 \mathrm{ppm}$. The dose was further reduced every 12 hours by $2-3 \mathrm{ppm}$ to a dose of $2 \mathrm{ppm}$ as long as there was no worsening of oxygenation. A trial of cessation of INO was then undertaken. A reduction of INO followed by an absolute decrease of $\mathrm{Spo}_{2}$ of $>10 \%$ or need for increase in $\mathrm{FIO}_{2}$ of $\geqslant 0.2$ to sustain the previous $\mathrm{SpO}_{2}$ reading within $\leqslant 30$ minutes of changing the INO dose was considered an indication of lack of tolerance to the change, and the prior dose was reinstituted. Treatment with INO was discontinued by seven days if no evidence of dependency was detected. If INO dependency had developed, INO was continued with repeated trials of discontinuation every 24-48 hours.

All patients were treated with assisted ventilation with time cycled, pressure limited, and/or patient triggered ventilation (Dräger Babylog 8000), or high frequency oscillatory ventilation (Sensormedics 3100A). Changes from one mode of ventilation to another were allowed, but were made in only one case between 3 and 72 hours. Tracheal aspirate fluid samples were collected by standard techniques for analysis of pulmonary inflammatory cytokines (interleukin $1 \beta$ (IL-1 $\beta$ ), IL-8); a marker of peroxidative injury (8-epi-prostaglandin $\mathrm{F}_{2 \alpha}$ (8-epi$\left.\mathrm{PGF}_{2 \alpha}\right)$ ); a proliferative and vasoconstrictor substance (endothelin 1 (ET-1)); and a marker of basement membrane injury (laminin). Samples were immediately frozen at $-70^{\circ} \mathrm{C}$ until batch analysis was carried out.

Blood methaemoglobin and inspired nitrogen dioxide were continually measured. Surveillance was conducted for haemorrhage in any organ, infection, thrombocytopenia, necrotising enterocolitis, and retinopathy of prematurity. Bloodstream infections were defined as clinical deterioration plus blood culture yielding a pathogenic organism. Pneumonia was defined as deterioration of respiratory status, concomitant with tracheal aspirate material yielding growth of a pathogenic organism. Cranial ultrasound examinations were performed before initiation of INO, during treatment if clinically indicated, and after discontinuation of INO therapy.

\section{Echocardiographic analysis}

Echocardiography was performed within the 48 hours preceding INO whenever possible. Elevation in pulmonary vascular resistance was considered to be present if there was a detectable tricuspid valve regurgitation jet.

\section{Respiratory monitoring}

The $\mathrm{FIO}_{2}$, ventilator pressures (peak inflating pressure and positive end expiratory pressure for patients on conventional ventilation, and mean airway pressure and amplitude for those on high frequency oscillatory ventilation (HFOV)), tidal volume, minute ventilation, and rates were recorded hourly. The $\mathrm{Spo}_{2}$ and the most recent blood gas tensions and $\mathrm{pH}$ were recorded. All variables were averaged from hourly readings for the six hours before for baseline measurements, and six hours before and six hours after the 24 and 72 hour time points.

\section{Tracheal aspirate analysis}

Tracheal aspirate samples were assayed for IL-1 $\beta$, IL-8, ET-1, and 8 -epi-PGF ${ }_{2 \alpha}$ utilising available enzyme linked immunoassay (ELISA) kits (Quantikine, R\&D Systems, Minneapolis, Minnesota). Laminin concentrations were assayed by competitive inhibition ELISA (Chemicon International, Temecula, California). The soluble secretory component of IgA (sSC-IgA) was assayed by methods established in our laboratory. ${ }^{4}$ All samples were assayed at least in duplicate and the results averaged. Coefficients of variation for each of these assays is $<10 \%$ in our laboratory.

\section{Statistical analysis}

Statistical testing for continuous variables was performed by paired $t$ test with Bonferroni correction for multiple comparisons. For non-parametric data comparison, Wilcoxon rank sign test was performed. Correlation coefficients were tested for significance by Spearman and/or Kendall tau beta tests and linear regression where appropriate. SPSS 8.0 statistical software was used for the statistical computations (SPSS Inc., Chicago, Illinois).

\section{RESULTS}

Patients

Trials were performed in 33 consecutive infants who met the criteria for entry. Twenty were male; 21 were black, 11 white, and one Hispanic. Mean birth weight was $736 \mathrm{~g}$ (range 509-1250 g); mean gestational age was 25.3 weeks (range 23-29); mean age at enrolment was 19 days (range 9-29). Twenty one infants were treated with antenatal steroids, 18 with antenatal magnesium sulphate; 14 were delivered by caesarean section and 29 received surfactant treatment.

\section{Pulmonary gas exchange}

All infants tolerated the initial three hour trial with evidence of improvement. However, two infants did not continue to receive INO beyond 48 hours as there was no evidence then of a decrease of $\mathrm{FIO}_{2}$ needs. For the remaining group of 31 infants, there was a significant reduction in $\mathrm{FIO}_{2}$ at 3 hours and at 72 hours of therapy (at which time the median dose of INO was $10 \mathrm{ppm}$ ) compared with baseline values $(\mathrm{p}<0.05$ for each comparison, paired $t$ test and Wilcoxon rank sign test). Mean $\mathrm{FIO}_{2}$ decreased from $0.75(0.23)$ to $0.63(0.22)$ at 3 hours and to $0.58(0.18)$ at 72 hours of INO therapy. The calculations include data on all 33 patients, including the two infants not receiving INO at 72 hours. The mean $\mathrm{FIO}_{2}$ at 72 hours was also lower than that at 3 hours ( $\mathrm{p}<0.05$, Wilcoxon rank sign test). The reduction in $\mathrm{FIO}_{2}$ occurred even as mean $\mathrm{Spo}_{2}$ showed an upward trend. The $\mathrm{Spo}_{2}$ was $90(6) \%$ at baseline, 91 (4)\% at 3 hours, and $93(5) \%$ at 72 hours. In spite of the reduced dose of INO by 72 hours, 14 of the 33 trials (45\%) resulted in an absolute reduction in $\mathrm{FIO}_{2}$ of $\geqslant 0.2$. Only five infants (including the two whose trial stopped before 72 hours) showed no change or an increase in $\mathrm{FIO}_{2}$ by 72 hours. Magnitude of reduction in $\mathrm{FIO}_{2}$ correlated inversely with the baseline $\mathrm{FiO}_{2} \quad(\mathrm{p}<0.05$; $r=-0.72$ ). The decrease in $\mathrm{FIO}_{2}$ showed no significant correlation with postnatal or gestational age, prior or concurrent usage of corticosteroids, antenatal corticosteroid use, gender, race, or presence of pulmonary air leak. The mean reduction in $\mathrm{FIO}_{2}$ was similar for infants treated with conventional mechanical ventilation (CMV) $(n=15)$ or $\operatorname{HFOV}(n=18)$ at baseline. For the subset of 15 infants with baseline $\mathrm{FiO}_{2} \geqslant 0.9$, the mean $\mathrm{FIO}_{2}$ at 72 hours had decreased to 0.68 (range 0.97$0.35)$.

Measurements of $\mathrm{tcPCO}_{2}$ during INO administration were unchanged. Tidal volume, minute ventilation, and mean airway pressure did not change significantly between initiating INO and 72 hours of administration, as the emphasis was to reduce $\mathrm{FIO}_{2}$. Mean airway pressure at baseline was 7.3 (1.1) $\mathrm{cm} \mathrm{H}_{2} \mathrm{O}$ for the CMV treated infants and $12.2(3.1) \mathrm{cm} \mathrm{H}_{2} \mathrm{O}$ for the HFOV treated infants. Only one patient underwent a change in type of assisted ventilation (from synchronised intermittent mandatory ventilation to HFOV) during the first 72 hours of $\mathrm{INO} \mathrm{FIO}_{2}$ needs did not change after initiation of HFOV.

Eight patients failed to tolerate the first attempt at discontinuation of INO at the end of their trials and required repeated attempts at discontinuation of the inhaled NO. The magnitude of initial response was not different in this group compared to that of the other 25 infants. The longest duration of treatment was 13 days.

Twenty one of 33 infants were treated with dexamethasone at the time of initiation of the INO trial. All had been receiving dexamethasone for more than 48 hours but still met entry criteria. There was no difference in mean decrease in $\mathrm{FIO}_{2}$ between the steroid pretreated group and those not using steroids. 

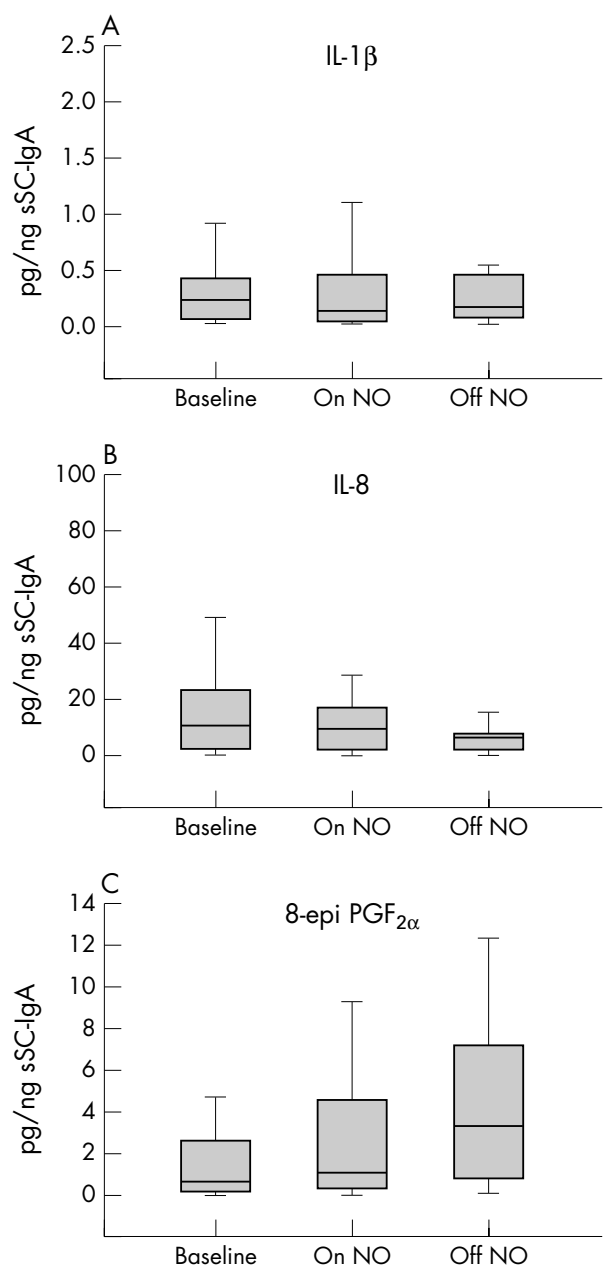

Figure 1 Boxplot of levels of (A) IL-1 $\beta$, (B) IL-8, and (C) 8-epi-PGF corrected for level of sSC-IgA before INO, after approximately 48 hours of INO, and after completion of INO treatment.

\section{Echocardiographic analysis}

Echocardiography was performed on 27 infants within 48 hours preceding initiation of treatment with INO. Eleven showed indirect evidence of increased pulmonary vascular resistance (PVR), in the form of a measurable tricuspid regurgitation jet. There was no correlation between the presence or absence of echocardiographic evidence of raised PVR and the magnitude of response to INO.

\section{serial tracheal aspirate sample analysis}

No difference was found in concentrations of IL-1 $\beta$, IL-8, 8-epi-PGF ${ }_{2 \alpha^{\prime}}$ ET- 1 , and laminin when values of each substance were compared between baseline and during INO or after INO therapy (figs 1, 2, and 3). There was wide variation in interpatient concentrations, but not in intrapatient concentrations, over time. When comparing concentrations across time in patients with a history of air leak (pulmonary interstitial emphysema or pneumothorax, $\mathrm{n}=5$ ), there again was no difference.

\section{Complications}

Five subjects showed intraventricular haemorrhages (IVH) of grade $3(n=3)$ or grade $4(n=2)$ at baseline. All other infants had grade 2 or less IVH prior to enrolment. No cases of new intracranial haemorrhage nor extension of old haemorrhage occurred. No subject experienced a clinically evident pulmonary haemorrhage. There were no sudden or unexplained decreases in haemoglobin or haematocrit, nor were

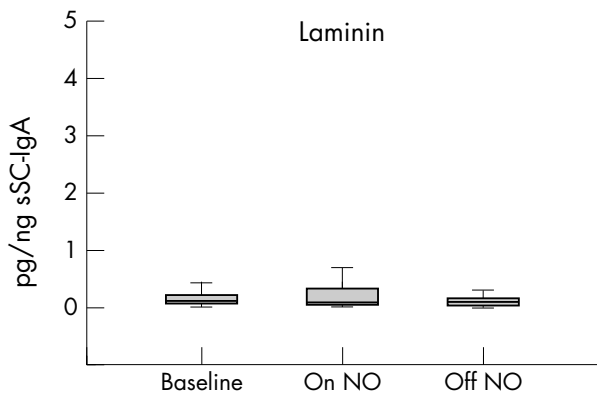

Figure 2 Boxplot of levels of laminin, corrected for level of sSC-lgA before INO, after approximately 48 hours of $\mathrm{INO}$, and after completion of INO treatment.

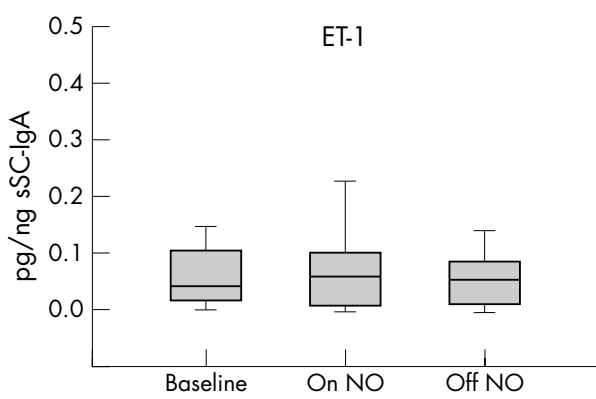

Figure 3 Boxplot of levels of ET-1, corrected for level of sSC-lgA before INO, after approximately 48 hours of INO, and after completion of INO treatment.

there any unexplained episodes of thrombocytopenia. No concentration of methaemoglobin above $2.8 \%$ was detected at any time during INO use. Bloodstream infection occurred in five patients (coagulase negative staphylococci in three, Candida sp. in two) during or following therapy with INO. Five patients developed pneumonia (ureaplasma/mycoplasma species in three, Candida sp. in one, and coagulase negative staphylococci in one) during or following INO.

\section{Outcome}

Four of the 33 infants died before discharge (see table 1). No additional infants died during mean period of 22 months (range 15-36). At 36 weeks postconceptional age (PCA), one child continued to be treated with assisted ventilation, two were treated with continuous positive airway pressure, and 22 were treated with nasal cannula supplemental oxygen. Three infants tolerated breathing room air. By 44 weeks PCA, only three infants remained hospitalised, two of whom were treated with nasal cannula oxygen; 10 infants were breathing room air, and 19 were utilising nasal cannula oxygen. At six months PCA, 25 infants had accessible records available for evaluation. Ten infants continued treatment with supplemental oxygen by nasal cannula; 15 were breathing room air.

\section{DISCUSSION}

Inhaled NO acutely improved pulmonary oxygen uptake in most infants with early CLD. This is the first report of administration of INO in premature infants without evidence of increasing airway inflammation, peroxidation, or basement membrane breakdown. We studied a specific but important population of infants. These extremely low birthweight infants had very significant but early CLD, probably characterised by substantial pulmonary inflammation. Use of INO has not been reported in this specific patient population, yet the pulmonary morbidity and mortality for $750 \mathrm{~g}$ infants still requiring assisted ventilation at 19 days of age with a mean $\mathrm{FIO}_{2}$ of 0.75 would be predicted to be considerable. 
Table 1 Details of the four infants who died

\begin{tabular}{|c|c|c|c|c|c|c|c|c|}
\hline Case & $B W(g)$ & $\begin{array}{l}\text { DOL at } \\
\text { admission } \\
\text { to } \mathrm{CMH}\end{array}$ & $\begin{array}{l}\text { DOL } \\
\text { at start } \\
\text { of INO }\end{array}$ & $\begin{array}{l}\text { Baseline } \\
\mathrm{F}_{2} / \mathrm{SpO}_{2}\end{array}$ & $\begin{array}{l}\text { INO } \\
\text { response } \\
\text { at } 72 \mathrm{~h}\end{array}$ & $\begin{array}{l}\text { INO } \\
\text { stopped, } \\
\text { DOL }\end{array}$ & $\begin{array}{l}\text { Age at } \\
\text { death } \\
\text { (days) }\end{array}$ & Associated problems \\
\hline 1 & 509 & 1 & 17 & $1.0 / 90 \%$ & Yes & 24 & 60 & \\
\hline 2 & 780 & 17 & 17 & $1.0 / 70 \%$ & Yes & 24 & 48 & $\begin{array}{l}\text { Steroid associated } \\
\text { cardiomyopathy from DOL } 10\end{array}$ \\
\hline 3 & 875 & 26 & 26 & $1.0 / 75 \%$ & Yes & 39 & 75 & Renal and hepatic failure \\
\hline 4 & 710 & 1 & 10 & $1.0 / 85 \%$ & No & 13 & 24 & Adenovirus pneumonia \\
\hline
\end{tabular}

Neonatal CLD appears to represent the effects of mechanical distortion of the lung, oxidative injury, and resultant inflammatory response in immature lungs. Evidence for early inflammatory changes in CLD has been documented. Increased concentrations of IL- $1 \beta$ and IL- 6 have been found in bronchoalveolar lavage fluid from patients with CLD, ${ }^{5}$ and IL- $1 \beta$ has been suggested as a participant in the development of CLD. ${ }^{67}$ The neutrophil chemotactic cytokine, IL-8, may participate in $C L D,{ }^{2}$ and both its production and that of IL- $1 \beta$ may be regulated directly or indirectly by $\mathrm{NO}^{8-11}$ and by peroxynitrites. $^{2}$ Our findings of no effect of INO on tracheal aspirate concentrations of IL-1 $\beta$ and IL-8 suggest that proinflammatory lung cytokine production was not worsened by INO administration. Inhaled NO could exert antiinflammatory effects by means of its effect on cytokines and neutrophil migration. Inhaled NO has also been shown to reduce neutrophil accumulation in the lung. ${ }^{31}$

There is evidence that 8 -isoprostanes such as 8 -epi-PGF ${ }_{2 \alpha \prime}$ which are generated by oxidation of membrane phospholipids, serve as markers for pulmonary oxidative injury, ${ }^{12}$ and may themselves contribute to pulmonary hypertension. ${ }^{13}$ Concentrations of the profibrotic and constrictor substance ET- 1 have been found to be increased in tracheal aspirate fluid of infants developing CLD. ${ }^{14}$ Tracheal aspirate concentrations of 8-epi-PGF ${ }_{2 \alpha}$ and ET- 1 were unchanged with NO treatment.

Concentrations of laminin, an indicator of basement membrane disruption, were also unchanged with use of INO in our patients. Thus, with relatively short term use of INO we found no evidence of additional peroxidative or inflammatory damage or of matrix disruption.

Microvascular obliteration and disorganised vasculogenesis in CLD may lead to pulmonary hypertension and contribute to poor matching of ventilation and perfusion, leading to hypoxaemia, hypercarbia, and exacerbation of pulmonary hypertension. Indirect evidence suggests that PVR is increased in patients who develop CLD. ${ }^{15}$ The pulmonary vasculature of premature infants can respond to nitric oxide with vasodilatation. ${ }^{16}$ Our results imply that in early CLD, INO may improve ventilation-perfusion matching by mechanisms including, but not limited to, reduction in PVR, as the response to INO did not depend on presence of raised PVR as measured by echocardiography. Some of the improvement in oxygenation may occur because NO may also function as a bronchodilator. ${ }^{17}$

Inhaled NO has been reported as a "rescue" therapy based on short term trials in preterm infants with severe RDS and hypoxaemic respiratory failure. ${ }^{16}$ Van Meurs and colleagues ${ }^{16}$ and Kinsella and colleagues ${ }^{19}$ tested the efficacy of NO in preterm infants beginning at $1-2$ days of age with total treatment duration of seven days in almost all infants. Subhedar and colleagues ${ }^{20}$ treated 10 infants with RDS from day 4 to day 7 with INO. As CLD may arise following mild or no previous RDS, these previously reported results do not predict the effect of INO on early CLD. Our study design also differs from that of Lonqvist and colleagues, ${ }^{21}$ who evaluated INO in nine infants with severe CLD by treating them for 40 minutes total, and from those of Banks and colleagues, ${ }^{22}$ who treated 16 infants with "end stage" CLD beginning at a median age of 3 months. Lonqvist and colleagues did not address safety issues, while Banks and colleagues did not measure directly markers of lung inflammation. No new intraventricular haemorrhage or extension of previous haemorrhage occurred in our study, in contrast to the results of Cheung and colleagues, ${ }^{18}$ in a study of the "rescue" use of INO in very premature infants treated at less than 24 hours of life. Cheung and colleagues found new haemorrhages or significant increases in extent of hemorrhage in 15 of 23 patients receiving INO who survived to undergo repeat ultrasound examination. During the time period encompassed by this study, we treated an additional six infants who were 2-14 months of age with INO for exacerbations of their underlying CLD. Our results were similar to those already well characterised by Banks and colleagues. ${ }^{22}$

Long term efficacy cannot be inferred from our study. We terminated NO usage, by design, at 7 days when possible, and had decreased the dose to $10 \mathrm{ppm}$ by 3 days. It is possible that a more substantial reduction in $\mathrm{FIO}_{2}$ could have been achieved with continuation of the starting dose of 20 ppm for 72 hours or more, or the same reduction achieved by starting at $10 \mathrm{ppm}$ without lowering the dose.

Chronic lung disease is now recognised as an independent contributor to poor neurodevelopmental outcome. ${ }^{23}$ In addition, diminished long term pulmonary function ${ }^{24}$ remains a major long term sequela of CLD. Our findings support the performance of a definitive trial of INO in early or evolving CLD, but do not offer sufficient support to warrant widespread use of INO in infants remaining on assisted ventilation for early CLD.

\section{ACKNOWLEDGEMENT}

This work was supported in part by NIH grant R-01-HL 58125 (WET). Portions of this work were presented at the Pediatric Academic Societies meeting, May 1999, San Francisco, California. We thank Steve Simon, PhD, for statistical assistance, Michael Norberg, MS, for analytical assistance, and the medical, respiratory therapy, and nursing staffs of the Intensive Care Nursery of the Children's Mercy Hospitals and Clinics.

\section{Authors' affiliations}

P L Clark, C A Castor, Section of Neonatology, Children's Mercy Hospitals and Clinics, 2401 Gillham Road, Kansas City, MO 64108 , USA

I I Ekekezie, H A Kaftan, W E Truog, Department of Pediatrics, University of Missouri-Kansas City School of Medicine, Kansas, USA

\section{REFERENCES}

1 Shenai JP. Vitamin A supplementation VLBW infants: rationale and evidence. Pediatrics 1999;104:1369-73.

2 Cuthbertson BH, Galley HF, Webster NR. The effects of nitric oxide and peroxynitrite on interleukin-8 and elastase from lipopolysaccharide-stimulated whole blood. Anesth Analg 1998;86:427-31.

3 Kinsella JP, Parker TA, Galan H, et al. Effects of inhaled nitric oxide on pulmonary edema and lung neutrophil accumulation in severe experimental hyaline membrane disease. Pediatr Res 1997:41:457-63.

4 Goil S, Barnes C, Thibeault D, et al. Simplified method for purification of colostrum to obtain secretory component of immunoglobulin A, using 
secretory component as a reference protein in tracheal aspirate fluid. $J$ Chromatogr B Biomed Sci Appl 1998;705:203-1 1 .

5 Kotecha S, Wilson L, Wangoo A, et al. Increase in interleukin (IL)-1 beto and IL-6 in bronchoalveolar lavage fluid obtained from infants with chronic lung disease of prematurity. Pediatr Res 1996:40:250-6.

6 Rindfleisch MS, Hasday JD, Taciak V, et al. Potential role of interleukin- 1 in the development of bronchopulmonary dysplasia. J Interferon Cytokine Res 1996; 16:365-73

7 Boota A, Zar H, Kim YM, et al. IL-1 beta stimulates superoxide and delayed peroxynitrite production by pulmonary vascular smooth muscle cells. Am J Physiol 1996;271:L932-8.

8 Villarete LH, Remick DG. Nitric oxide regulation of interleukin-8 gene expression. Shock 1997;7:29-35.

9 Kuo HP, Hwang KH, Lin HC, et al. Effect of endogenous nitric oxide on tumour necrosis factor-alpha-induced leukosequestration and IL-8 release in guinea-pigs airways in vivo. Br J Pharmacol 1997;122:103-11.

10 Thomassen MJ, Buhrow LT, Connors M, et al. Nitric oxide inhibits inflammatory cytokine production by human alveolar macrophages. Am J Respir Cell Mol Biol 1997; 17:279-83.

11 Collet-Martin S, Gatecel C, Kermarrec N, et al. Alveolar neutrophil functions and cytokine levels inpatients with the adult respiratory distress syndrome during nitric oxide inhalation. Am J Respir Crit Care Med 1996; 153:985-90

12 Goil S, Truog WE, Barnes C, et al. Eight-epi-PGF2alpha: a possible marker of lipid peroxidation in term infants with severe pulmonary disease. J Pediatr 1998;132:349-51.

13 Truog WE, Norberg M, Thibeault DW. Effects of 8-epi-prostaglandin F2 alpha and $U 46,619$ on pulmonary hemodynamics in piglets. Biol Neonate 1997:71:306-16.

14 Niu JO, Munski UK, Siddig MM, et al. Early increase in endothelin-1 in tracheal aspirates of preterm infants: correlation with bronchopulmonary dysplasia. J Pediatr 1998;132:965-70.
15 Benatar A, Clarke J, Silverman M. Pulmonary hypertension in infants with chronic lung disease: non-invasive evaluation and short-term effect of oxygen treatment. Arch Dis Child Fetal Neonatal Ed 1995;72:F14-19.

16 Van Meurs KP, Rhine WD, Asselin JM, et al. Response of premature infants with severe respiratory failure to inhaled nitric oxide. Preemie NO Collaborative Group. Pediatr Pulmonol 1997;24:319-23.

17 Potter CF, Dreshaj IA, Haxhiu MA, et al. Effect of exogenous and endogenous nitric oxide on the airway and tissue components of lung resistance in the newborn piglet. Pediatr Res 1997;41:886-91.

18 Cheung PY, Peliowski A, Robertson CM. The outcome of very low birth weight neonates $(=1500 \mathrm{~g})$ rescued by inhaled nitric oxide: neurodevelopment in early childhood. J Pediatr 1998;133:735-9.

19 Kinsella JP, Walsh WF, Bose CL, et al. Inhaled nitric oxide in premature neonates with hypoxemic respiratory failure: a randomized controlled trial. Lancet 1999;354:1061-5.

20 Subhedar NV, Ryan SW, Shaw NJ. Open randomized controlled trial of inhaled nitric oxide and early dexamethasone in high risk preterm infants. Arch Dis Child Fetal Neonatal Ed 1997;77:F185-90.

21 Lonqvist PA, Jonsson B, Winberg $P$, et al. Inhaled nitric oxide in infants with developing or established chronic lung disease. Acta Paediatr 1995;84: 1188-92

22 Banks BA, Seri I, Ischiropoulos $\mathrm{H}$, et al. Changes in oxygenation with inhaled nitric oxide in severe bronchopulmonary dysplasia. Pediatrics 1999;103:610-18.

23 Singer L, Yamashita T, Lilien L, et al. A longitudinal study of developmental outcome of infants with bronchopulmonary dysplasia and very low birth weight. Pediatrics 1997;100:987-93.

24 Jacob SV, Coates AL, Lands LC, et al. Long-term pulmonary sequelae of severe bronchopulmonary dysplasia. J Pediatr 1998;133:193-200.

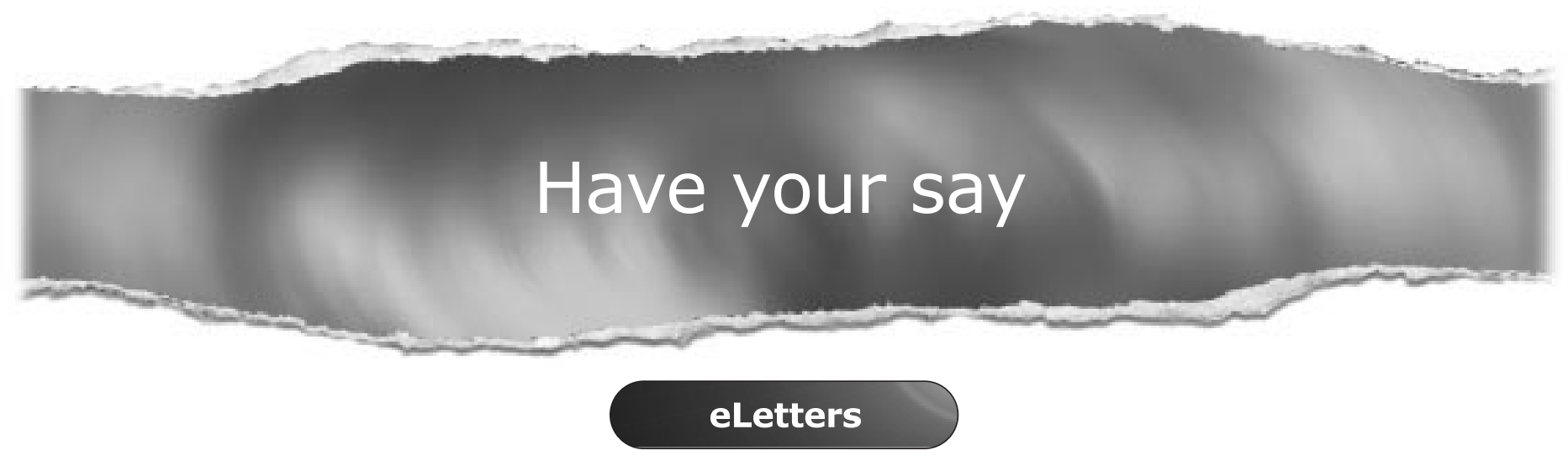

If you wish to comment on any article published in Archives of Disease in Childhood you can send an eLetter using the eLetters link at the beginning of each article. Your response will be posted on Archives of Disease in Childhood online within a few days of receipt (subject to editorial screening).

\section{www.archdischild.com}

